\title{
Écrire et parler. Quelques préalables théoriques
}

\section{Rudolf Mahrer}

\section{OpenEdition}

\section{Journals}

Édition électronique

URL : http://journals.openedition.org/genesis/1371

DOI : 10.4000/genesis. 1371

ISSN : 2268-1590

\section{Éditeur :}

Presses universitaires de Paris Sorbonne (PUPS), Société internationale de génétique artistique littéraire et scientifique (SIGALES)

\section{Édition imprimée}

Date de publication : 17 novembre 2014

Pagination : $29-49$

ISBN : 9782840509714

ISSN : 1167-5101

Référence électronique

Rudolf Mahrer, «Écrire et parler. Quelques préalables théoriques », Genesis [En ligne], 39 | 2014, mis en ligne le 12 décembre 2016, consulté le 19 avril 2019. URL : http://journals.openedition.org/ genesis/1371 ; DOl : 10.4000/genesis.1371 


\section{Écrire et parler. Quelques préalables théoriques}

Rudolf Mahrer

$\mathrm{U}$

ne génétique de la performance orale fait tôt ce constat : souvent, en amont d'une parole, l'écriture a été requise. Les documents qui en témoignent appartiennent à la famille des activités de préparation. Quel que soit le domaine considéré (sport, musique, architecture, littérature, cuisine, ingénierie...) et le type de rituel préparatoire (par exercices comme l'entraînement des sportifs ou la répétition des musiciens ou par production de documents comme la maquette des architectes ou le prototype des ingénieurs...), lorsqu'on s'intéresse aux modes de préparation, il convient de comparer les propriétés du procès ou de l'objet en vue (le préparé) et les propriétés des procès ou objets de la préparation elle-même (le préparant). Par ce biais seulement, il est possible de comprendre pourquoi et comment le préparant sert le préparé, en l'occurrence, pourquoi et comment la trace écrite sert la performance orale. Pourquoi en somme l'orateur se fait-il scripteur le temps, je veux dire l'espace de la préparation?

Ajoutons encore que l'examen sémiologique de la question suppose quelques hypothèses, d'ordre génétique, sur ce que préparer veut dire, et voilà délimitée notre contribution à la réflexion collective du volume. Ce que l'oral doit à sa préparation écrite mais aussi ce qu'il ne lui doit pas - impose de penser ce qui caractérise l'un et l'autre. La question est aussi rebattue que résistante. Y revenir sous la forme de quelques préalables sémiologiques aura, espérons-nous, des vertus heuristiques.

Écrire n'est pas la seule chose qu'on puisse faire pour préparer un discours - on peut aussi herboriser -, mais c'est une chose qu'on fait souvent. L'étude des paroles singulières dans l'histoire de leur élaboration rencontre donc cet objet paradoxal d'un avant-dire qui est un écrit produit dans l'horizon d'un dire qui est un oral. On ne saurait réfléchir sur ces documents étranges sans prendre tout d'abord acte de l'irréductible différence qui oppose les signaux graphiques et les signaux acoustiques. Cette donnée empirique évidente mais fondamentale explique les difficultés qu'éprouvent toutes les tentatives théoriques se proposant d'embrasser les discours par-delà la substance qui les réalise et les deux systèmes de signes, oral et écrit, qui les informent.

L'écrit et l'oral : propriété des signaux 
Les brouillons écrits des textes à finalité orale peuvent apparaître dès lors comme un corpus particulièrement approprié à la réflexion sur les relations entre l'écrit et l'oral. Après tout, les propriétés formelles de l'oral spontané lui-même n'ont-elles pas été rapprochées de celles des brouillons de l'écrit (Blanche-Benveniste et Jeanjean 1987, p. 150-162) ? Et, inversement, c'est le terme d'écrit qui vient à l'esprit quand il s'agit d'analyser les productions orales non spontanées obéissant à des protocoles formels établis. C'est par exemple le cas de Joëlle Gardes Tamine qui, voulant caractériser les genres du discours oraux où l'on rencontre des appositions nominales, parle d'abord de « discours technique », « discours public », « discours professionnels », « situations de "protocole" » pour résumer finalement ainsi : «des situations d'oral écrit, si l'on peut dire » (Gardes Tamine 2004, p. 151).

Derrière une telle caractérisation métalangagière, ce qui est visé, ce n'est pas l'écrit du système ou l'écrit de la substance, mais celui d'une grammaire ; celle-ci n'est pas réservée à l'écrit mais s'y trouve préférentiellement représentée (Blanche-Benveniste 1990, Gadet 1996, Béguelin 1998, Berrendonner 2004...). On préfère d'ailleurs aujourd'hui considérer que cette grammaire est caractéristique non d'un médium, mais de certaines pratiques discursives, dites de la « distance communicative » (Koch et Oesterreicher 2001).

Située strictement sur le plan grammatical (ou segmental), l'analyse conduit à une conception « continuiste » de la relation entre scripturalité et oralité. En effet, tout ce qui se dit à l'oral peut se dire à l'écrit et inversement (Rey-Debove 1988, Blanche-Benveniste et Jeanjean 1987, Béguelin 1998...). Mais par un trop fort rapprochement, on perd plus que l'on ne gagne, qu'il s'agisse d'analyser tant l'écrit oralisé du roman que l'oral transcrit de la linguistique de corpus ou encore, précisément, les avant-dire écrits des interventions orales. Nous partirons d'une logique inverse, qui consiste à scruter l'écart pour appréhender mieux ensuite comment, en dépit de leurs différences ou plutôt en raison d'elles, l'écrit sert l'oral : celui de l'orateur, du plaideur, de l'enseignant... Sans y faire référence de manière toujours explicite, les quelques réflexions sémiologiques qui vont suivre ont ce genre de productions comme horizon d'application.

\section{L'écrit et l'oral, l'espace et le temps}

L'examen de la substance qu'informent les systèmes de signes doit d'abord retenir l'attention du sémiologue (Benveniste 2012 [1969] et Harris 1993).

Le discours oral présente à cet égard deux propriétés fondamentales : il est unidimensionnel et processuel. Sous l'angle matériel, il est doté de propriétés acoustiques, mais, plus fondamentalement encore, de propriétés chronologiques. La syntagmatique de la parole orale n'a qu'une dimension, et c'est pour cette raison que lui sied, à elle seule, l'image du «canal ». Chaque partie y est repérable selon une coordonnée temporelle, relativement à un tout doté d'une durée mesurable. Encore que cette description n'est vraie que d'un point de vue rétrospectif : car le tout du discours oral n'est jamais présent et disponible en tant que tel ; il ne peut donc se mesurer qu'après coup. Les parties constituant le signal acoustique étant successives, elles ne nous sont pas données ensemble, mais suivant l'axe orienté et irréversible du temps. D'où l'apparent paradoxe : l'oral, comme signal, a une durée, mais, faute de support, il n'est pas durable. Dès lors, la perception du 
signal impose la présence de l'auditeur au moment même de l'émission. Le fait ne manque pas d'avoir toutes sortes de conséquences « interactionnelles » bien connues.

En tant que signal, l'oral réclame un volume, celui du liquide qu'il met en mouvement. Mais il signifie par ce mouvement, sans se fixer dans le volume. Ce n'est pas une empreinte ou une trace qui sert de support à l'activité sémiotique, mais le flux continu des transformations produites par l'émission sonore sur son environnement. Le terme d'environnement dénote ici - en amont de toute considération sur la cognition et l'énonciation constructive de l'environnement - le milieu, physique et social, où prend place l'activité sémiologique. On peut dès lors distinguer, du point de vue de l'espace, énonciation orale et énonciation écrite, en affirmant que seule la seconde requiert un support, en plus d'un environnement (Anis 1998, p. 16).

Le rapport à l'espace du signal acoustique à la base de l'énonciation orale se réduit à celui qu'il entretient avec l'environnement où il se produit. Sa circulation est une propagation, toujours en rapport direct avec sa source ; elle n'est déterminée que par les qualités acoustiques intrinsèques du signal (notamment son intensité), certaines propriétés de son environnement (comme son relief) et la concurrence d'autres bruits. Le signal est par conséquent indissociable de son site d'émission. Pour s'y soustraire, il requiert une technologie annexe d'enregistrement. Or l'enregistrement, c'est une trace gravée sur un support ; ce n'est donc plus en lui-même un signal acoustique, processuel. Caporossi et Leblay, qui étudient les processus d'écriture (plutôt que leurs traces), proposent justement de remplacer l'expression de «temps réel » par celle de "temps enregistré » (Caporossi et Leblay 2014, p. 11-12). Cette dernière expression fait mieux apparaître ce qu'implique l'analyse de tout processus : une opération de stabilisation qui transforme fondamentalement l'objet en question. L'analyse des objets émanant des sémiologies de la trace ne suppose pas une telle altération. L'écrit est nativement trace.

L'écrit justement. La lecture lui assure sa signifiance. En tant qu'activité, la lecture se développe dans le temps, selon des rythmes qui sont ceux du lecteur. Il en va tout autrement du support empirique dont la lecture est actualisation : la substance de l'expression écrite ou signal graphique. Les parties de la trace graphique n'entretiennent aucune relation chronologique objective les unes avec les autres : elles sont matériellement simultanées et donc toutes disponibles en même temps. Seule l'émission de la trace graphique, c'està-dire l'écriture, s'organise selon une chronologie. Inférer le processus à partir de son produit écrit, tel est d'ailleurs le geste interprétatif définitoire de la critique génétique (Lebrave 1983), geste qui, pour spécifique qu'il soit à cette discipline parmi les sciences du langage, n'en fait pas moins partie de l'activité interprétative ordinaire des scripteurs et des lecteurs (Mahrer 2009). Ce point de vue sur l'écrit consiste à reconstruire ou à modéliser un processus, l'écriture, mais sa donnée n'en est pas moins la trace écrite, statique. Lorsqu'elle traite de documents déjà écrits, la génétique s'appuie donc sur (et contribue à) une sémiologie de l'écrit (et non pas de l'écriture) qui interroge celui-ci comme un indice de son processus d'engendrement.

Le repérage interne à l'objet graphique - les relations mutuelles de ses parties - s'opère donc non pas sur l'axe temporel mais selon des coordonnées spatiales. Paradoxalement, bien que l'écrit dure, il constitue un signal sans durée, en ce sens que, entre son début et sa fin, c'est-à-dire ses points de départ et d'arrivée conventionnels, ce n'est pas un temps 
qui s'écoule, mais un champ spatial qui s'ouvre, une étendue. Le signal écrit en tant que tel se donne sans déroulement. Toutes les parties d'un signal graphique sont synchrones : elles se donnent en même temps sur l'espace délimité de leur support. Durable mais sans déroulement, telles sont les qualités qui font l'écrit «monumental » au sens de Barthes : « c'est la parole, éphémère, qui est indélébile, non l'écriture, monumentale » (1971, p. 887). Lorsque Dominique Combe déclare : "À l'écrit, contrairement à ce que pourrait suggérer le paradoxe signalé par Barthes, la main ne se contente pas de raturer, de corriger ou d'effacer, c'est-à-dire de substituer une expression à une autre : l'écriture, comme la voix, "bredouille" » (2002, p. 15-16), le poéticien, clairement, passe de l'écrit à l'écriture, déplace la réflexion de Barthes pour confondre, comme beaucoup, propriété du signal et propriété de son émission. Sur le plan de la donnée empirique, le caractère synchrone des parties du discours écrit a pour conséquence que le temps de la lecture n'est pas édicté par celui de l'émission du signal, ni par celui de la mémoire de cette émission. Le processus de l'interprétation n'est pas contraint par le déploiement du signal dans le temps, mais par son déploiement plus ou moins durable dans l'espace. Le temps de la compréhension offert par l'écrit est celui qu'on peut et veut bien lui accorder. Ses limites « objectives » sont celles de l'accès au support et de la conservation de celui-ci. L'écrit donne ainsi au verbal les conditions d'interprétation qui sont celles du tableau peint.

\section{Des déterminations empiriques aux discours}

Par où, dès lors, commencer la comparaison de deux systèmes de signes si distincts par la nature de leurs signifiants? Nous avancerons dans l'analyse des rapports sémiologiques entre oralité et écriture en intégrant, toujours sous l'angle de la substance, les paramètres de l'énonciation. Si l'on admet que, sous cet angle, l'action symbolique consiste en l'échange de signaux, dans un environnement donné, entre des instances d'émission et de réception (qui peuvent être la même personne), alors l'analyse portera sur le rapport entre le signal et i) son émetteur, ii) son environnement et iii) son récepteur. Il s'agit autrement dit d'intégrer les «facteurs inaliénables de la communication verbale »(Jakobson 1963, p. 214) à l'exclusion du code, qui n'est pas un fait de substance, et moyennant la prise en compte du « message » en tant que substance. Il s'agit, autrement dit encore, de décrire comment les signaux - selon leur nature empirique et la manière dont celle-ci régit leurs relations à l'espace et au temps - pèsent sur les propriétés des discours.

[1] Comme processus sans support, le signal oral est lié à l'espace de son émission, physique d'abord, social ensuite dès lors que le signal est perçu comme tel par un co-énonciateur et sert de base à une interaction symbolique. Or ce qui lie le signal à son environnement, c'est le corps du locuteur. Pour cette raison, le signal acoustique de la communication orale entretient une relation particulière avec ses circonstances d'émission.

Ordinairement, le signal acoustique s'accompagne d'autres sémiologies processuelles extralinguistiques, dites "kinésiques » ou « mimogestuelles », que le corps humain est capable de produire et de percevoir ; le plus souvent culturellement construites, ces sémiologies sont spontanément convoquées dans l'oral informel, éventuellement anticipées dans l'oral formel. Cette complémentarité, liée à la nécessaire présence de l'émetteur et 


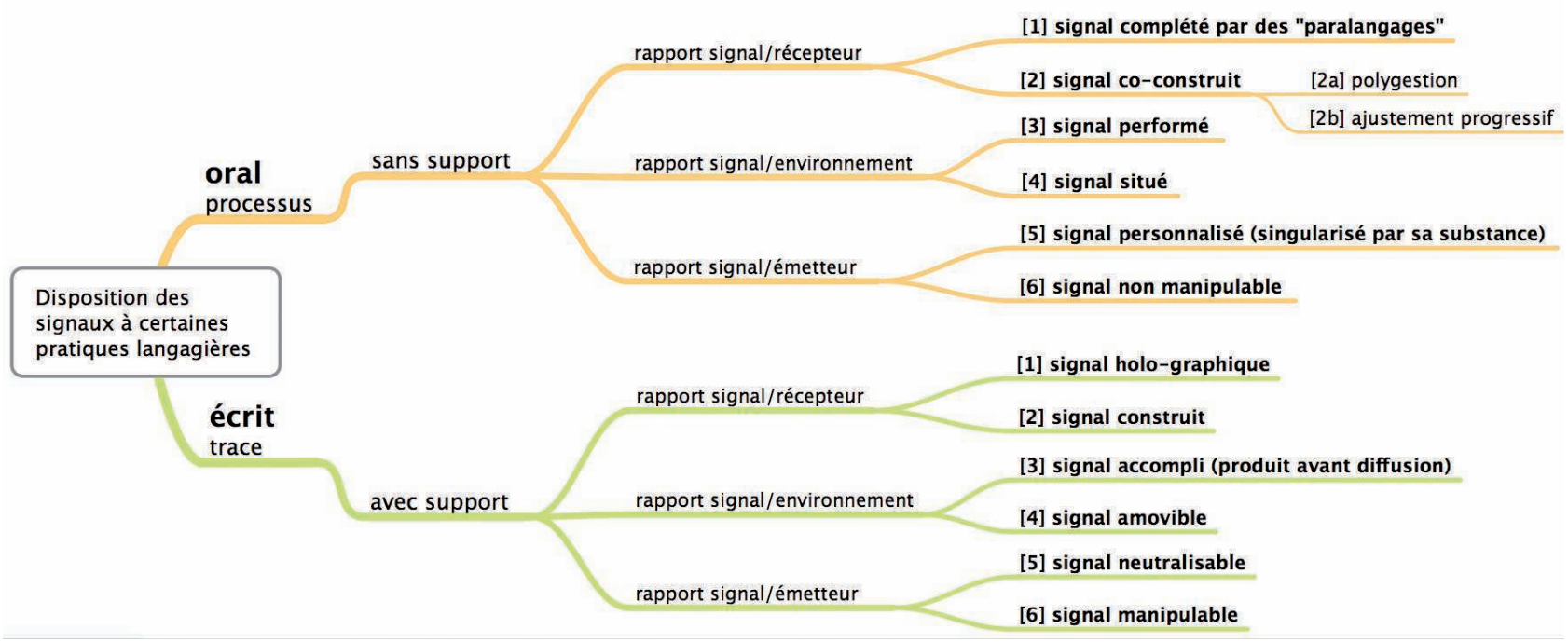

Schéma 1 : Affordance des signaux (manière dont ils disposent à leur usage)

de son corps - et donc toujours à l'absence de support - est parfois considérée comme définitoire de la « parole totale » (Rey-Debove 1988, p. 81).

[2] En situation orale, la coprésence des émetteurs ne manque pas d'influer sur l'élaboration du signal lui-même, qui peut être polyphonique au sens le plus acoustique du terme. Même dans le cas de l'oral formel, le récepteur est un émetteur potentiel, non contrôlé par l'émetteur actuel, et l'émission peut ainsi se réaliser à plusieurs. Le co-énonciateur est à tout moment susceptible de compléter, chevaucher, interrompre... un signal en cours d'émission : l'orateur le plus imperturbable tient compte des marques d'approbation, d'ennui, etc. qu'il reçoit du public (le «back-channel ») comme il est contraint de faire un sort aux interventions ou demandes d'interventions verbales plus ou moins prévues par l'activité. En conséquence, l'action que permet ce signal se conceptualise préférentiellement comme un échange, une interaction. Le caractère spécifique de cet «échange » ne tient pas à l'ajustement préalable du signal à l'action symbolique qu'il vise, mais bien au réglage effectué au fil de l'émission des signaux. Cette accommodation permet de réviser l'action programmée dans le cours de son accomplissement, révision qui peut toucher tous les aspects de l'émission, des plus substantiels (parler moins rapidement, plus fort...) au plus sémantiques (parler moins durement, moins techniquement...).

À l'opposé, le signal graphique interpose un écran, entre la situation d'émission et la situation de réception : le rapport direct entre les interlocuteurs n'est pas interdit, mais il n'est plus obligatoire. De ce possible détachement du corps de l'émetteur et de son environnement d'émission découle la majeure partie des caractéristiques associées 
à l'énonciation orale et, distinctivement, à l'énonciation écrite. Sur l'axe énonciateurénonciataire, ce qui caractérise l'écrit, c'est la possibilité d'une énonciation sans récepteur présent au moment de la production du signal. Cette possibilité en conditionne une autre : celle d'une situation de production préalable à la situation de réception. La possible disjonction entre production et réception ménage une coulisse à l'énonciation : le développement historique de pratiques backstage explique l'imaginaire de l'écriture dans nos sociétés à forte littératie : on la pense intime en tant que processus mais publique en tant que produit.

Publique «essentiellement » et de manière « irréductible » (Harris 1993, p. 147), la communication écrite le serait en vertu de la rémanence de son signal, caractère effectivement définitoire des sémiologies de la trace. L'utilisation "intime » de l'écrit n'est bien sûr pas impossible ; tout au plus constitue-t-elle, selon Harris, une "perversion » du potentiel de l'écrit, réclamant la mise au point de dispositifs compensatoires d'« intimisation » (ibid.) comme l'enveloppe. De la même façon, l'oral formel non dialogal sera considéré comme « contre-nature ». L'oral trop léché risque de s'assimiler à de l'écrit lu : moins une performance qu'un produit, moins un échange (voire une communion) qu'un don. Trop public, il peut passer pour impersonnel. Un orateur préparé n'en vaut pas nécessairement deux.

En somme, l'oral, situé et co-construit, est actio et interactio. L'écrit de préparation d'oral intégrera peut-être cette dimension en incluant des instructions relatives au comportement langagier à adopter en fonction des réactions de l'auditoire, verbales ou paraverbales. La note de la leçon probatoire ou de l'entretien d'embauche préparera peut-être des réponses à des questions attendues, la note de cours signalera des parties à « sauter » selon les connaissances variables des étudiants ou le temps restant à tel moment de la performance didactique.

[3] Sur le plan de la conduite langagière, la coïncidence dans la durée entre émission (manifestation) et réception définit le régime performanciel de l'oral. Parce qu'il est une performance - même si celle-ci a été préparée - d'autres émissions et d'autres événements lui sont concomitants, le concurrencent et peuvent l'interrompre. Dès lors, la performance du signal oral n'est pas totalement prévisible. Sur le plan linguistique, ce facteur réduit la pertinence d'une planification textuelle totale ; il affecte également l'intégration, syntaxique et textuelle, des énoncés (phrases bien formées, progression thématique continue, cohésion anaphorique, isotopie et isoplasmie, plans de texte clairs, etc.). Mais on trouve aussi dans le régime performanciel de l'énonciation orale l'occasion d'une valorisation : celle de la spontanéité, de la sincérité, du naturel... que révélerait l'« oral authentique ». Il arrive ainsi étonnamment que l'oral qui apparaît tout à fait planifié, prémédité, calculé... perde en crédibilité.

Pour sa part, le signal-trace, par définition, ne peut constituer une performance. Faute de durée, il ne saurait se dérouler en même temps que quoi que ce soit. Le signal graphique lui-même n'est pas une opération mais un produit auquel le récepteur s'expose et qu'il actualise. Dès lors, stricto sensu, on ne peut parler de trace ou d'écrit improvisé. Bien sûr, l'écriture peut être " performée », c'est-à-dire produite sous les yeux de ses lecteurs. Le geste, processuel, recèle bien une sémiotique propre dont on pourrait entreprendre 
l'analyse. Mais il s'agirait alors d'une sémiotique de l'écriture, et non pas d'une sémiotique de l'écrit. Or ce qu'analyse la linguistique de l'écrit et ce qu'élabore le lecteur, c'est la signifiance de l'écrit et non celle de l'écriture. Le caractère accompli de l'écrit disjoint le geste de sa production (traçage du signal) et celui de sa mise en circulation. Entre ces deux gestes différents et successifs pour l'écrit, le scripteur a la possibilité d'accomplir (éventuellement plusieurs fois) le processus d'autoréception et les éventuelles réécritures qui peuvent en découler.

Enfin, la gestion de la performance suppose celle des émotions liées aux enjeux de l'action sociale engagée. Le caractère performanciel fonde également la présence, dans les discours oraux, de marqueurs et d'indices d'émotion, et de constructions jugées expressives - souvent sans autre argument d'ailleurs que leur forte présence à l'oral et leur moindre présence à l'écrit : certains types de phrases averbales, les constructions disloquées ou clivées, les phrases à présentatif...

En perdant sa dimension performancielle - non sans une nostalgie souvent manifeste - le signal graphique gagne la qualité que l'on place souvent à l'origine de l'invention de l'écriture : celle de stockage, de mémoire externe, d'archive (Goody 1977) ; c'est d'évidence le cas pour l'avant-dire, qui est appelé, le moment venu, à changer de substance. À partir de là, on comprendra aisément que la capacité à gérer la performance soit fortement valorisée dans l'évaluation des compétences communicationnelles orales et, inversement, peu ou non pertinente pour l'écrit. Une part importante des discussions entourant la célèbre anaphore rhétorique de François Hollande, « Moi, président de la République » (syntagme repris quinze fois de suite, lors du débat télévisé du 2 mai 2012) consistait à évaluer le caractère improvisé ou prémédité du procédé. Une telle évaluation ne change rien à la performance elle-même, mais elle a un enjeu très manifeste sur son appréciation. Hollande lui-même a défendu le caractère partiellement spontané de son anaphore, alors qu'un article du Point paru le 10 mai 2012 documentait sa préparation. La discussion touche au rapport ambigu de ce genre de pratique discursive formelle ou semi-formelle avec l'oralité et l'écriture (paraître spontanée pour une performance orale en réalité optimalement planifiée par l'écrit). Elle touche surtout à une échelle de valeurs parmi les qualités présidentiables : celles de l'oralité (spontanéité, réactivité, vitesse, émotivité, subjectivité) ou celles de l'écriture (préparation, anticipation, profondeur, rationalité, élaboration à plusieurs). Selon qu'on l'interprète comme écrite puis oralisée ou comme "authentiquement » orale, la même performance confère à son auteur tantôt l'ethos du bon élève, sérieux, peut-être calculateur, voire ridicule si l'on ne goûte pas la figure, tantôt elle taille le costume de l'homme sincère, transporté par ses émotions, éventuellement jusqu'à l'excès.

Reconnaître que l'oral est performance, quel que soit le degré de sa préparation - même quand le « texte » a été appris par cœur, comme au théâtre -, c'est reconnaître qu'il ne connaît pas de bon à tirer. Il n'y a pas de forme arrêtée, prête à la reproduction, sur laquelle la qualité de la performance finale puisse être tout à fait gagée. Une performance ne se reproduit pas. Du passage de l'écrit à l'oral, ou du passage d'un oral à un autre oral présentant la même structure segmentale, il y a à chaque fois une nouvelle réalisation dans un temps nouveau. La réécoute (ou réénonciation orale) implique une réémission de signal, la relecture (ou réénonciation écrite) non. Pour cette raison, la dernière étape d'une genèse 
écrite d'un discours oral peut se tenir à une importante distance segmentale du discours qu'il a préparé1.

[4] Dans le rapport étroit qu'il entretient avec le corps de l'émetteur, le signal acoustique est lié à son environnement d'émission. La verbalisation tendant à s'appuyer sur des données contextuelles accessibles perceptivement, l'oral est par suite le lieu d'un fonctionnement et d'un développement exophoriques du langage. L'écrit, en tant que trace, dispose au transport - ce n'est bien sûr pas son unique mode d'être (enseigne, signalisation, graffiti, etc.), mais c'en est un qui compte dans l'histoire et dans l'état actuel de notre société. Amovible, il est contraint à une autosuffisance "référentielle », à un travail de cohésion endophorique plus intense ainsi qu'à un travail d'explicitation de son contexte (physique, social et pragmatique). L'écrit pour l'oral profite évidemment de cette transportabilité, celle de la note qui accompagne l'orateur sur les lieux de ses performances. Mais cette différence est aussi une contrainte pour le causeur qui recourt au papier : l'écrit est préparation « hors situation » d'une action située.

[5] Si l'énonciation orale ne peut échapper « à la pointe du hic et nunc », comme l'écrit Lebrave (1992, p. 36), cela n'est vrai que de l'émission du signal, et non de sa conception, qui toujours s'opère en amont - de quelques centièmes de seconde pour l'oral «spontané » à plusieurs années pour l'oral "préparé ». De la contemporanéité du signal et de sa réception, il ne faut donc pas conclure que le discours oral ne puisse être préparé en quelque façon ${ }^{2}$.

L'oral formel (qui donne lieu à une genèse écrite) et l'oral spontané ne s'opposent pas simplement comme l'élaboré au non élaboré : mais le premier ajoute des traces à la nécessaire genèse mentale de tout discours. De fait, l'oral à préparation écrite suppose une « seconde » préparation, endophasique cette fois, au moment d'être prononcé.

De la non-coïncidence temporelle entre l'émission de la parole et sa conception témoignent, par exemple, de nombreuses gloses du type " $\mathrm{j}$ 'allais dire $\mathrm{x}$ », « j'ai failli dire $\mathrm{x}$ », « j'ai dit $\mathrm{x}$, je voulais dire $\mathrm{y} » .$. Sans minimiser la dimension rhétorique de ces formules, chacun peut éprouver en son for le déphasage qu'elles révèlent, au sein des discours les plus spontanés, entre ce qu'on projette de dire et le propos qu'on tient en effet quelques fractions de seconde plus tard. Quant à cette part rhétorique, elle consiste notamment à conférer une allure spontanée aux phrases les mieux préméditées - par un jeu plus ou moins habile de gloses et de reformulations (« J'ai trouvé votre remarque, comment dirais-je, audacieuse », "Votre discours, j'allais dire votre harangue, nous a pris aux tripes... », etc.).

1. Par segmental, on entend le niveau de structuration d'un signal qui est partagé par le système écrit et le système oral (phonème/graphème, morphème, syntagme et catégorème). Ces systèmes comportent par ailleurs des modules de structuration spécifiques, dits « suprasegmentaux » qui sont la prosodie pour l'oral, la topographie (ponctuation) pour l'écrit. Une étude approfondie de la manière dont l'écrit aide à préparer l'oral réclame de décrire en quoi le dessin (topographie) sert la mélodie (prosodie).

2. Ce serait confondre la dimension performancielle de l'oral avec son éventuel caractère improvisé. Cette dernière concerne la contemporanéité de la conception du signal et de son émission alors que la performance signifie la contemporanéité de l'émission et de la réception. 
La dimension de performance de l'oral, au sens anglais et récent d'« œuvre présentée dans le cours de sa réalisation » (Robert), est, en toute rigueur, à restreindre à son signal. Ce n'est pas tout l'oral, mais ce n'est pas rien non plus : ce que les Anciens appelaient actio est une opération suffisamment coûteuse et complexe pour générer des accidents que, sur le fil du hic et nunc, l'oral, indélébile, ne saura pas cacher. L'écrit consistant pour sa part en une trace préalablement performée, il souffre en comparaison d'un déficit « performanciel » que certaines poétiques chercheront à combler ${ }^{3}$. Son émission elle-même est une activité d'atelier, comme la peinture (quand elle ne se fait pas «performance »), et une activité secondaire au point qu'on puisse en confier la responsabilité à autrui (le prote, le typographe). Dans l'oral public, par défaut, celui qui émet la parole passe pour l'avoir produite, qu'il l'ait inventée ou non, il habite la parole de sa voix. Dans l'écrit public, c'està-dire majoritairement imprimé, le présupposé est inverse : le signal, neutralisé dans sa substance par la typographie, n'est pas un facteur de subjectivation.

[6] L'absence de support n'instaure pas seulement une relation d'immédiateté entre émetteur et signal, elle détermine également la manière dont le signal, comme objet empirique, s'offre à la manipulation. Notons tout d'abord que toute activité de manipulation d'un signal linguistique déjà émis sera considérée comme métalinguistique. Mais cette activité se réalise de manière radicalement différente selon que le signal est acoustique et sans trace, ou qu'il est graphique, donc accompli et rémanent. Rappelons aussi qu'un signal acoustique peut laisser une trace, s'il est enregistré. Toutefois, si le signal est reçu lui-même, en tant qu'il est processus acoustique et non en tant qu'inscription sur un support (enregistrement), alors il ne laisse de trace que dans la mémoire : seul son souvenir peut être manipulé. La modification du déjà-émis est impossible. À ce sujet, construisant la spécificité de leur objet, les linguistes de la production écrite que sont les généticiens rappellent volontiers l'incipit du «Bruissement de la langue » de Roland Barthes :

La parole est irréversible, telle est sa fatalité. Ce qui a été dit ne peut se reprendre, sauf à s'augmenter : corriger, c'est, ici, bizarrement, ajouter. En parlant, je ne puis jamais gommer, effacer, annuler ; tout ce que je puis faire, c'est de dire «j'annule, j'efface, je rectifie », bref de parler encore. Cette très singulière annulation par ajout, je l'appellerai bredouillement (Barthes 1975, p. 800).

Dire sur son dire - redire ou dédire -, telle est l'activité méta-énonciative à laquelle contraint le signal acoustique. À l'impossible réécriture du signal, l'énonciation orale supplée par une activité réflexive dont la visée n'est pas de modifier le signal lui-même, mais ses effets. L'énonciation orale n'a d'autres choix que de placer «vers l'avant » ses « retours en arrière », pour reprendre la métaphore spatiale de Rey-Debove, de BlancheBenveniste et de la tradition, c'est-à-dire d'installer les réanalyses du déjà-émis dans la successivité de l'émission, dans l'omniprésence du processus - non pas toujours présent, mais toujours au présent. La méta-énonciation qui rejoint le signal dans le fil de son émission participe nécessairement de l'énonciation. On perçoit ici tout le bénéfice de distinguer le plan substantiel de l'analyse sémiologique : au niveau des formes toujours

3. Voir sur ce point l'article de Gilles Philippe, supra. 
relatives à leur sens, la méta-énonciation modalise ou modifie le déjà-dit ; mais la réfection orale est impossible sur le plan de la substance et laisse derrière elle intact le signal déjà émis.

La rémanence du signal graphique favorise au contraire sa manipulation par l'énonciateur-scripteur. Relativement à la structure autodialogique de l'activité énonciative, on peut dire que la substance graphique survit à la durée du cycle de l'autodialogisme, par lequel l'énonciateur pourvoit son signal de formes et de sens, et s'offre aux modifications éventuelles que cette assignation, nécessairement rétroactive, lui paraît appeler. Ces manipulations de la substance graphique sont analysées en quatre types d'opération (ajout, suppression, remplacement et déplacement) constitutives de l'activité de réécriture (voir par exemple Grésillon, Lebrave et Viollet 1990). La fabrication du signal à part soi qui précède son émission pour l'autre permet au scripteur de conserver ou d'éliminer les traces des « retours en arrière », des biffures situées par-dessus, par-dessous, à la place de ou ailleurs. La critique génétique qui prend pour objet cette fabrication de l'écrit est une discipline sans équivalent dans le domaine des sémiologies processuelles. Linguistique et génétique de l'oral se confondraient dans la mesure où coïncident interprétation du signal acoustique et observation du processus qui le fait advenir.

Cette possibilité de se retourner sur son dire-écrire est variablement exploitée selon les situations et les pratiques de l'écrit (l'examen écrit, l'écriture diariste, la minute n'offrent pas la même liberté de réécriture que d'autres genres) ; en outre, le scripteur est libre de n'en point user. À l'inverse, nous le savons, certaines paroles et certains genres oraux appuient leur émission sur une préparation écrite et une mémorisation. Il demeure qu'une possibilité est offerte à l'écrit, par la qualité du signal qu'il requiert, déterminant les pratiques discursives qui vont, elles-mêmes, déterminer des produits spécifiés. De fait, la manière, diamétralement opposée, dont les signaux acoustiques et graphiques disposent à leur révision est le plus souvent considérée sous l'angle de la correction ou de l'ajustement qu'ils rendent possibles.

L'écriture manuscrite se corrige directement vers l'arrière, et l'écriture neutralisée [l'imprimé] fait disparaître les traces des corrections ; au contraire, le discours parlé ne peut rien faire vers l'arrière, sinon commenter métalinguistiquement ses erreurs ou imprécisions, en les corrigeant par un nouveau discours vers l'avant (Rey-Debove 1988, p. 85).

La manière dont la qualité du signal affecte sa possible ou son impossible manipulation détermine l'attente, à l'écrit, d'énoncés cohérents, complets, corrects et recourant aux variantes grammaticales hautes. Au plan de la forme, cette propriété du signal explique que l'énonciation écrite ait été longtemps considérée comme le modèle syntaxique unique à l'aune duquel devait être jugé l'ensemble des productions. Manipulable, il est perfectible. L'ensemble des accidents envisagés plus haut, liés au régime performanciel de l'oral (interruption ou manifestation du récepteur, irruption d'événements contextuels, difficulté à gérer la complexité de la tâche, émotion non contrôlée) sont sans fondement empirique à l'écrit. Répétitions (lexicales ou syntaxiques), faux départs, structures incomplètes, reformulations paraphrastiques, etc., seront pour cette raison considérés comme des phénomènes caractéristiques de l'énonciation orale (Blanche-Benveniste 1990, p. 17-29). Parce que les traces de l'activité méta-énonciative sont ineffaçables à l'oral, leur 
présence est considérée comme indice d'oralité. À l'écrit ces traces sont potentiellement escamotables par la technique de la rature, donnant au discours ainsi préparé l'aspect d'un discours « spontanément définitif » (Lebrave 1987, t. I, p. 44, n. 15).

Enfin, la rémanence de la trace permet à l'écrit de disjoindre deux gestes conjoints à l'oral : celui de l'émission et celui de la communication. Le second vient valider le premier, d'où la valeur «entérinée » qu'on reconnaît à l'écrit. En effet, entre ces deux gestes, le signal lui-même (et non seulement le sens) s'offre à la révision - permettant aux cycles de l'autodialogisme d'ajuster par retouches successives intention et effet sur soi. Dès lors l'écrit se voit conférer un degré élevé de prise en charge énonciative. Le discours oral (performé, en situation, potentiellement perturbé par les émotions ou des facteurs externes, et non manipulable) est moins commissif que le discours écrit qui passe, par défaut, pour réfléchi - au sens propre - et donc devant être pleinement assumé. Selon son propos et ses visées, l'oral à genèse écrite tentera de se situer entre ces deux bornes, soit du côté de la spontanéité, de l'émotivité ou de l'authenticité de l'oral, soit de celui du calcul, de la rationalité et de l'élaboration de l'écrit.

À cette disponibilité pour la manipulation (disponibilité diversement exploitée selon les traditions discursives et les individus), ajoutons la profonde différence de rapport au temps et à l'espace, et l'on a réuni les facteurs principaux qui expliquent l'usage de la métaphore du fil pour représenter l'oral et celle du tissu, désormais lexicalisée, pour représenter l'écrit. On comprend pourquoi la parole, qui est un processus, résiste, dans le sens commun au moins, à être envisagé comme textus, malgré les invitations répétées d'influents linguistes.

À présent qu'ont été passés en revue les effets de la nature des signaux sur la communication (effets récapitulés par le schéma de la page 33), revenons au rapport très différent qu'écrit et oral entretiennent avec le temps et l'espace. Car c'est à partir de ce rapport que s'explique comment l'écrit sert la préparation de l'oral.

Ce n'est pas le moindre problème de la tradition saussurienne que la temporalité de la parole soit abordée par la notion de « linéarité ». Si le terme n'est pas de Saussure ${ }^{4}$, il est reçu depuis le Cours de linguistique générale comme le « second principe » du signe linguistique, après son caractère arbitraire. La « linéarité du signifiant » est bien sûr une métaphore spatiale ; on sait l'espace plus facile à appréhender que le temps. Ce dont il est ici question à proprement parler, c'est de successivité. Aussi Saussure considère-t-il que l'articulation du langage oral, c'est-à-dire notre capacité à distinguer des parties et à les agencer en touts mémorisables, repose sur l'analyse de la chaîne acoustique dans le temps. À l'écrit, seule la divisibilité dans l'espace confère au signal graphique un pouvoir de distinction et de mémorisation équivalent. Si l'écrit, par sa spatialité, « représente » l'oral de manière optimale, ce n'est pas parce que le temps est idéalement figuré par une ligne, mais parce que, sur le plan cognitif et sémiologique, seul le séquençage spatial peut remplir une fonction analogue pour l'œil à celle du séquençage temporel pour l'oreille.

4. Grâce au travail de Testenoire (2010), nous savons que le terme de « linéarité » ne fut pas choisi par Saussure lui-même ; il n'apparaît pas dans les notes manuscrites.

\section{L'irréductibilité de l'écrit à une ligne}


La prémisse bancale selon laquelle le signifiant phonique serait linéaire conduit le linguiste à identifier le linguistique au linéaire. Ce qui du scriptural déborde de la ligne est du coup renvoyé à d'autres sémiologies. Est proprement linguistique ce qui est convertible dans la successivité d'un signal acoustique, c'est-à-dire lisible au sens d'oralisable. Cela revient à ne prendre en considération que l'axe horizontal de l'objet écrit. Cette ablation du vertical, les éditeurs en sont coutumiers, et tout particulièrement l'éditeur des notes prises en vue d'interventions orales 5 :

[...] il est symptomatique que même lorsqu'ils s'attaquent à des brouillons, nombre d'éditeurs optent pour une présentation linéarisée des additions, suppressions et substitutions que comportent les manuscrits : ce qui dans l'original est pluridimensionnel et polymorphe est aplati sur la ligne et ramené à la norme unidimensionnelle caractéristique du texte (Lebrave 1992, p. 67).

La successivité du signifiant, figurée par la forme d'une ligne, fonde toute l'analyse de la langue en différents « niveaux » linguistiques. On comprendra qu'elle ait la peau dure et que, même sous le terme problématique de "linéarité », on la situe au cœur de l'analyse linguistique, y compris quand il s'agit d'écrit.

C'est par exemple le traitement théorique que réserve aux manuscrits de travail Lebrave, sans doute le généticien le plus soucieux d'expliciter les présupposés linguistiques de sa discipline : "Si l'on admet que l'écrit transpose l'enchânement temporel des séquences phoniques de l'oral en une séquence linéaire de symboles graphiques, un texte écrit est fondamentalement uni-dimensionnel » (Lebrave 1983, p. 14). Il réserve dès lors la bidimensionnalité aux seuls brouillons :

[La] linéarité est mise en cause dans un brouillon par le supplément de données qui vient s'ajouter à la « ligne graphique » et qui donne à la page une seconde dimension. Cette dimension supplémentaire est particulièrement claire dans les corrections interlinéaires, où l'on « voit » le premier segment écrit, sa biffure, et le segment écrit au-dessus ou au-dessous qui le remplace (Lebrave 1983, p. 14).

Or, bien qu'il exploite l'espace graphique à sa façon, moins standardisée que ne l'est celle de l'imprimé, le brouillon n'est pas opposable à l'imprimé du point de vue du nombre de ses dimensions. L'opposition fondatrice de l'avant-texte et du texte ne peut se concevoir comme une différence dimensionnelle : tous les écrits «mettent en cause » le principe de la linéarité du signifiant linguistique.

L'identification du linguistique au lisible, du lisible à l'oralisable et de l'oralisable au linéaire conduit à écarter la possibilité d'une syntagmatique verticale ${ }^{6}$. Or ce qu'une

5. La récente édition des notes prises par Jean-Paul Sartre en vue de conférences prononcées au Havre pendant l'hiver 1932-1933 a par exemple fait le choix de procurer une « version de lecture courante » de ce document, en donnant au long les mots et noms abrégés, en reconstituant des paragraphes que l'original ne donnait pas, et en éliminant bien sûr tacitement tous les codes graphiques utilisés par l'orateur (dir. A. Cohen-Solal et G. Philippe, Études sartriennes, $\mathrm{n}^{\circ} 16,2012$ ). Publier consiste toujours à créer un nouvel espace de signification.

6. On discutera ailleurs la façon dont la notion de « paradigmes de réécriture », courante en critique génétique pour décrire les formes « inscrit[es] verticalement sur le flux horizontal de la linéarité syntagmatique d'un avant-texte » (Grésillon 1994, p. 245), m'apparaît comme une stratégie théorique pour sauvegarder la « linéarité » du signifiant en réduisant la verticalité de l'écrit à une manifestation accidentelle et temporaire de l'axe des substitutions. 
linguistique de l'écrit doit décrire, ce sont précisément les types de prédications qui peuvent être réalisés dans la verticalité. Les concepts pour penser la dimension spatiale du langage écrit ne sont pas nouveaux (on pense par exemple à la vilisibilité d'Anis, 1983, ou à l'iconotexte de Montandon, 1990). Il ne faut pas en réduire la portée : ils ne doivent pas signifier la possible mise en espace de l'écrit, mais bien que l'écrit est constitutivement espace.

$\mathrm{Si}$, opposant la successivité temporelle de la substance acoustique à l'espace bidimensionnel de la substance graphique, on prend la mesure de l'hétérogénéité radicale qui distingue les signaux dont une même langue est forme, alors nous ne serons plus tentés (comme nous l'étions par la fallacieuse métaphore spatiale de la ligne) d'identifier le système écrit au système oral. Nous irons au contraire vers une sémiolinguistique pensant l'autonomie des deux systèmes et, partant, leur interaction au sein d'un même idiome. Avant même d'interroger les relations que ces deux langues dans une langue pourront bien entretenir, il faut accueillir la possibilité d'un énoncé écrit qui soit un objet spatial à côté d'un énoncé oral qui soit un objet processuel. En faisant place à cette hétérogénéité des signaux, on en fera également une à la linguistique de l'écrit. Celle-ci ne poursuivra pas son élaboration solide sur des métaphores empruntant à l'oral des concepts impropres à décrire son champ d'expérience, qui est visuo-graphique. Qui a jamais observé les documents préparant une intervention orale ou la transcription d'une telle intervention sait bien qu'ils ne se donnent en rien comme l'avers ou le revers d'une même médaille linguistique : ils sont tout autre chose, un espace préparant un temps.

La segmentation en blocs graphiques (titres, listes, paragraphes, chapitres...) opère un jeu de positions relatives, horizontales et verticales. Dans la sémiotique de la notation mathématique, la barre de fraction et les complexités de syntagmation qu'elle offre, ou, dans le domaine de l'imprimé, l'agencement des réponses du questionnaire/formulaire à choix multiples sont autant de dispositifs spatiaux qui imposent de penser la manière dont les discours écrits investissent les rapports positionnels sur deux axes et la manière dont la langue écrite les règle. Celle-ci a développé un sous-système qui lui est propre, appelé communément ponctuation, dont la fonction est de gérer l'espace graphique, horizontalement, c'est-à-dire dans les lignes, et verticalement, et donc ouvrant à la page. Dans une ponctuation ainsi définie, il faut donc inclure les signes noirs mais aussi la gestion du blanc (Favriaud 2011).

Considérons ainsi cet extrait des notes manuscrites prises par Jean-Paul Sartre en 1933 en vue d'une conférence sur John Dos Passos ${ }^{7}$ :

\begin{tabular}{l|l} 
Les ouvriers & $\begin{array}{l}\text { Joe } \\
\text { Mac }\end{array}$ \\
& $\begin{array}{l}\text { Charly : l'ouvrier sans opinion définie } \\
\text { Ben Compton : le révolutionnaire }\end{array}$
\end{tabular}

7. On trouve le contexte et les références de cette citation supra, p. 17. 
On observera la fonction des deux accolades (le trait vertical en tient toujours lieu chez Sartre, il peut d'ailleurs présenter un léger renflement central). Elles ont valeur de marqueurs d'intégration verticale (répondant aux " marqueurs d'intégration linéaire » de Coltier et Turco 1988). La première signale que le nom commun, à gauche, est un hyperthème que les quatre noms propres, à droite, viennent saturer. La procédure descriptive, dite d'aspectualisation, n'est pas ici l'effet d'un verbe, ni d'organisateurs (« le premier », «l'un/l'autre », « et »...), mais celui de la seule verticalité, assistée par la fonction conventionnelle de l'accolade. Si on l'analyse plus avant, on relèvera que l'aspectualisation résulte d'une coordination implicite réalisée sur l'axe vertical et d'une prédication attributive réalisée sur l'axe horizontal. Par la combinaison des deux axes, la ligne, ouverte par la majuscule du déterminant, se ramifie ; le chemin bifurque d'une manière inconnue pour le locuteur et l'auditeur'.

Le «paradigme nominal » ainsi constitué ne réclame aucune marque de clôture : sa spatialité synchrone (le fait que toutes ses composantes soient simultanément disponibles à notre vue) suffit à en signaler les limites. La seconde accolade signifie un mouvement sémantique inverse, de résomption, selon un dispositif dont seul l'ordre change (symétrie axiale). Les deux premiers prédicats de l'accolade la plus longue sont joints dans un prédicat nouveau attribué en commun. Si on comparait cet énoncé graphique avec quelques-unes de ses oralisations paraphrastiques acceptables ${ }^{9}$, on relèverait aussi que par leur position centrale (axe horizontal) et par leur empilement (axe vertical), les noms Joe et Mac s'enchâssent dans les deux structures prédicatives décrites (aspectualisante et résomptive), une fois sujet, une fois prédicat, sans qu'il soit nécessaire ni de les répéter, ni de les anaphoriser. On aura beau faire, on ne réalisera jamais ce double emboîtement à l'oral. Il est un fait de spatialité.

L'espace ainsi décrit est déjà une verbalisation. Mais dans le contexte d'un écrit préparant l'oral, il n'est encore qu'une oralisation en puissance : reste encore à le transformer en processus. Chaque axe a une portée sémantique propre, directement perceptible par la vision et qui va soutenir l'oralisation. Il prépare l'actio, mais ne stocke pas l'expression exacte que l'orateur va/veut proférer.

L'exemple nous permet de penser simplement cet apparent paradoxe : l'espace des notes comporte de nombreuses données qui ne sont pas oralisables telles quelles, alors même que le support et son apport écrit sont ici au service d'une oralisation à venir. Dès qu'il s'écarte un tant soit peu de l'alignement de caractères - de ce moderne boustrophédon auquel le sens commun réduit l'espace graphique - l'écrit fait en réalité autre chose que de stocker les unités du discours à « performer ». Peut-être plus encore que ses brouillons préparant un écrit, les notes prises par Roland Barthes en vue de ses cours recèlent par exemple des diagrammes,

8. L'appel-renvoi de note (Lefebvre 2011) est un dispositif spécifiquement graphique reposant sur la bidimensionnalité de l'espace graphique et ajoutant à la bifurcation décrite ici une rupture : par l'appel-renvoi de note, la ligne se prolonge ici et ailleurs, imposant au lecteur un choix dans l'ordre de son activité sémiotique et sémantique. Un tel choix d'ordre est inexistant à l'oral.

9. «Les ouvriers étaient quatre : Joe, Mac, Charly et Ben Compton. Les deux premiers étaient aussi des aventuriers. » (Il serait ici plus rigoureux de recourir à une transcription phonétique qui n'introduirait pas de nouveaux interprétants spatiaux.) 
des listes et des signes algébriques 10 ; de soigneuses notes marginales (notamment les références paginées) semblent anticiper de possibles questions, etc. L'espace graphique de la note peut parfois s'apparenter à un support pour l'oralisation, soit un espace préformaté pour son transcodage en séquence temporelle. Ce n'est manifestement pas le cas des notes de Barthes. Certains critiques relèvent d'ailleurs l'extrême « esthétisation de la page » de ses manuscrits de cours ${ }^{11}$. La page est un espace de signifiance qui va préparer et servir l'activité de l'orateur, il s'agit de décrire de quelle manière, schématiquement.

Si l'on s'intéresse à l'activité de préparation elle-même, il convient de ne pas réduire ses produits aux étapes d'un processus aboutissant à l'état ultime et achevé12. Pour une linguistique des textes de préparation (voir Mahrer et Nicollier 2014), les documents génétiques s'apparentent davantage à des moyens et des techniques mis en œuvre afin d'atteindre un but. La nuance n'est pas négligeable : on peut certes considérer la maquette comme un «stade » de la voiture, le plan comme une « étape » de la maison, l'entraînement du sportif comme un «état » de la performance. Mais alors voiture, maison et performance sont envisagées comme des projets incluant leur préparation et non en eux-mêmes : un plan n'est pas une maison, il est éventuellement appréhendable en tant qu'étape de la construction de la maison et non en tant qu'étape de la maison. Surtout, si l'on veut considérer l'objet préparatoire en lui-même - étudier la sémiotique du plan architectural pour la comprendre, l'enseigner ou la rénover - il faut y voir autre chose qu'une maison in posse : un produit culturel spécifique avec des propriétés et des fonctions qui ne sont pas celles du bâtiment. Si l'on admet, pour prendre un autre exemple encore, que la recette de cuisine est un objet culturel, c'est bien qu'on a préalablement considéré ses propres caractéristiques discursives (Greimas 1983, Vigner 1990, Adam 2001a et b), plutôt que les propriétés, chimiques ou gustatives, du plat qu'elle aide à préparer. La recette est l'objet du sémiologue autant que de l'historien de la cuisine.

Faisons de même pour les technologies, graphiques pour certaines d'entre elles au moins, qui servent à préparer un discours oral : considérons-les dans leurs propriétés intrinsèques. À présent que nous avons caractérisé schématiquement la nature des moyens préparatoires et celle de l'objet préparé, nous pouvons avancer quelques hypothèses conclusives sur l'intérêt de celle-ci pour celui-là.

Si on laisse désormais de côté les différences de substance qui distinguent radicalement les discours oraux et les discours écrits, on peut envisager ces derniers ensemble selon la partition de la rhétorique traditionnelle. Produire un discours, écrit ou oral, suppose

\section{Préparer \\ la performance orale}

10. Voir, par exemple, ici même dans la rubrique Inédit, le cours de Barthes reproduit ; on notera que Roland Barthes réfléchit alors à une articulation des dimensions temporelle et spatiale du langage.

11. Thomas Clerc (préface à Roland Barthes, Le Neutre, Paris, Éditions du Seuil/IMEC, 2002, p. 16), cité dans Bellon (2012, p. 62).

12. Notons que dans cette approche traditionnelle, on tend à juger l'état $x+1$ comme plus achevé que 1 'état $x$, sans bien savoir si ce jugement se fonde sur des qualités intrinsèque de $x+1$ ou simplement sur le fait que ce dernier se situe sur l'axe temporel plus proche de l'état ultime.

L'espace de la préparation du discours 
l'inventio, la dispositio et l'elocutio ; en outre, l'oral suppose encore la maitrise de l'actio, soit la performance du signal (quand l'écrit, on l'a dit, tolère que la production du signal soit déléguée à une autre instance). Selon ce schématisme, dont la valeur heuristique est antique et vénérable, produire un discours combine en somme la faculté de choisirfabriquer des contenus et des expressions linguistiques, et d'en déterminer l'ordre. On distinguera trois manières pour l'espace de préparer la performance orale : 1) l'espace heuristique (inventio et dispositio), 2) l'espace de la formulation (elocutio) et 3) l'espace de la préperformance (actio et inter-actio).

1. L'espace heuristique. Les qualités matérielles de l'écrit (la durabilité et la coprésence des signaux graphiques sur leur support $v s$ la successivité des signaux acoustiques) disposent i) à la segmentation visuo-spatiale des constituants linguistiques et textuels et ii) à la représentation globale de l'énoncé. Ces deux dispositions confèrent à l'écrit un fort pouvoir organisateur. Pour en rendre compte, les recherches en psychologie cognitive supposent l'existence, distincte de la mémoire verbale, d'une mémoire visuo-spatiale « requise en production écrite lorsque le rédacteur organise le contenu de son texte » (Olive, Passerault, Le Bigot, Lebrave 2010, p. 39). L'espace heuristique de l'écrit de préparation « utilis[e] des procédés visuo-spatiaux pour hiérarchiser l'information (par exemple, des diagrammes) » (p. 38-39), ceux-ci sollicitant la mémoire correspondante pour « permettre aux rédacteurs de se construire une carte conceptuelle du contenu de leur texte » (p. 38).

Ainsi l'espace heuristique est-il au service de l'organisation (dispositio) de l'énonciation qu'il prépare. Mais cette valeur ne va pas seule. D'autres travaux de psycholinguistes (notamment ceux de Galbraith et alii 2005) confirment l'intuition pédagogique selon laquelle les plans prérédactionnels - mises en espace des formes linguistiques en vue d'ordonnancer le discours - stimulent l'invention des contenus. L'espace heuristique profite tout à la fois à la dispositio et à l'inventio.

Un brouillon d'oral exploitant l'espace de la page pour la découverte et l'ordonnancement se distingue faiblement d'un brouillon d'écrit. Il est certes, in fine, un écrit pour parler, mais dans le processus d'élaboration, considérée de plus près, il est d'abord un écrit préparant un autre écrit. Et au bout de la chaîne seulement se situe une parole. La genèse écrite des discours oraux pose en effet toujours cette difficile question : le document de la genèse que nous avons retrouvé dans l'archive prépare-t-il la performance orale elle-même, ou un état antérieur de celle-ci ? Ces notes que nous étudions sont-elles celles que l'orateur avait sous les yeux au moment de discourir ou des notes préparant d'autres notes pour le discours ? Les discours oraux aussi peuvent avoir des dossiers génétiques complexes.

2. L'espace de la formulation. Les qualités du signal graphique disposent encore, on l'a dit, à la réélaboration des constituants linguistiques et textuels. L'espace de la formulation est celui que le scripteur déploie au profit de la recherche de l'expression du discours préparé. Il se caractérise par la présence des traces de la réécriture venant, selon différentes modalités spatiales et matérielles, intercepter une ligne graphique, pour y insérer, substituer, supprimer ou déplacer de nouvelles séquences linguistiques ; mais il peut comporter aussi des prédications métascripturales réfléchissant l'état de la formulation et instruisant des reformulations encore à venir. Sa topographie est donc reconnaissable : ce n'est plus celle 
du plan qui délimite la page en zones entre lesquelles des fléchages ou des numérotations instruisent un parcours ; l'espace de la formulation est principalement ligné, mais porte des biffures, minimes ou massives, et des greffes de toute taille qui forment des ramifications locales.

En tant que la grammaire préférentielle des genres oraux n'est pas la même que celle des genres écrits, on pourrait reconnaître l'espace de la formulation d'un écrit de préparation d'oral ( $v s$ un écrit de préparation d'écrit) selon des critères grammaticaux. Néanmoins, les différences morphosyntaxiques entre écrit et oral s'estompant dans les genres oraux « de la distance communicationnelle » (précisément ceux qui souvent se préparent par écrit), cette spécification morphosyntaxique risque d'être ténue et dépendra du genre du discours oral préparé.

3. L'espace de la préperformance. En tant que l'actio est émission d'un signal, elle touche à la substance et nous renvoie à nos premières remarques : l'écrit partage avec l'oral les formes d'expression et de contenu qui constituent le plan segmental de sa structuration, mais il ne partage pas sa substance. Dès lors, l'écrit ne peut stocker l'actio, il ne peut que la préparer ${ }^{13}$. L'espace de la préperformance connaît par suite deux modes de préparation de l'oralisation.

3.1 Selon un fonctionnement intersémiotique, des symboles graphiques instruisent la conversion, ou transcodage, de l'écrit en oral : il s'agit de topogrammes liés à valeur prosodique (soulignements divers), mais aussi d'instructions intersémiotiques, comme " être bref/rapide/court sur ce point », « 2 minutes [pour cette partie/page] », « s'arrêter/ insister/glisser sur cette question »..., thématisant, avec une valeur instructionnelle plus ou moins marquée, la performance visée, dans ses dimensions propres, acoustiques et temporelles. Les didascalies du texte théâtral - genre d'écrit pour parler s'il en est ressortissent de cet espace de la préparation de l'actio.

3.2 Une autre sémiotique graphique prépare indirectement l'oralisation : elle vise à faciliter l'opération de lecture simultanée à la production du signal. Par exemple, du point de vue des formes linguistiques, la note sera dépouillée pour ne présenter à la vue du conférencier qu'un viatique essentiel à son parcours : les composantes minimales d'un raisonnement, les étapes d'un récit, les parties d'un objet d'étude, etc. C'était le cas de la note de Sartre commentée plus haut. La topographie (gestion de l'espace de la page) et le recours à différents topogrammes liés plus ou moins conventionnels (soulignement, surlignage, cerclage de couleur...) peuvent venir aider le repérage visuel sur la page des noyaux thématiques ou des articulations du propos.

Dans le premier cas (3.1), il s'agit de se donner des consignes pour la constitution du signal lui-même : l'écrit instruit l'oralisation directement. Dans le second cas (3.2), il s'agit de minimiser le coût de la lecture (ou de la remémorisation) pour que l'effort cognitif soit essentiellement tourné vers l'oralisation.

13. Dans Mahrer et Nicollier 2014, nous distinguons quatre modalités de la préparation écrite du discours : la programmation, l'organisation, l'invention et l'archivage. 
Déchiffrer un manuscrit ou suivre ses notes
[...] on peut dire, en appelant « objets » spatiaux les différents fragments d'écriture que le brouillon comporte, que la lecture des manuscrits suppose préalablement la description de la position relative de ces objets les uns par rapport aux autres (Lebrave 1983, p. 14).

Ce que Lebrave dit du brouillon vaut pour l'écrit gravé, imprimé, numérique... Cela vaut avec d'importantes différences d'intensité selon les genres du discours (l'affiche, la poésie, les écrits journalistiques...), mais avec une inflexible identité de principe ${ }^{14}$.

L'interprétation de la topographie confine aux plus fastidieux décodages lorsqu'il s'agit de suivre l'entrelacs des formulations d'un écrivain comme Flaubert ou Proust. Mais, inversement, la signifiance spatiale est la béquille graphique, le guide-âne précieux du parleur qui a tracé son chemin sur une carte.

Tirer du rapport positionnel des unités écrites un sens qui aide à la performance orale, telle est l'opération commune au généticien et à l'orateur qui «suit ses notes 15 ». Et si la signifiance sémantique (au sens de Benveniste) s'inscrit principalement dans la syntagmatique horizontale de l'écrit, soit la distribution des unités sur la chaîne, les regroupements qu'opère l'intuition syntaxique (et que ne manifestent pas les seuls rapports de contiguïté) peuvent également répondre, à l'écrit, d'une syntagmatique verticale. Ces deux syntagmatiques organisent la page en un espace de signifiance qui va préparer l'activité de l'orateur. Gageons que l'ensemble de ces remarques théoriques nous aidera à comprendre de quelle manière.

\section{Références bibliographiques}

Adam Jean-Michel (2001a), « Types de textes ou genres de discours? Comment classer les textes qui disent de et comment faire?», Langages, $\mathrm{n}^{\circ} 141$, p. 10-27.

- 2001b, «Entre conseil et consigne : les genres de l'incitation à l'action », Pratiques, $\mathrm{n}^{\circ} 111-112$, p. 7-38.

ANIS Jacques (1983), «Vilisibilité du texte poétique », Langue française, n 59, p. 88-102.

- 1998, Texte et ordinateur. L'écriture réinventée ?, Paris-Bruxelles, De Boeck.

BARTHEs Roland (2002 [1971]), «Écrivains, intellectuels, professeurs », dans CEuvres complètes, t. III (1968-1971), Paris, Éditions du Seuil, p. 887-907.

— 2002 [1975], « Le bruissement de la langue », dans CEuvres complètes, t. IV (1972-1976), Paris, Éditions du Seuil, p. 800-803.

BÉGUelin Marie-José (1998), « Le rapport écrit-oral. Tendances dissimulatrices, tendances assimilatrices », Cahiers de linguistique française, $\mathrm{n}^{\circ} 20$, p. 229-253.

Bellon Guillaume (2012), Une parole inquiète. Barthes et Foucault au Collège de France, Grenoble, ELLUG.

14. Il ne s'agit pas de nier les spécificités sémiotiques et sémantiques des manuscrits de travail, mais de les situer au sein d'une sémiologie de l'écrit qui accorde une juste place à l'espace de la langue écrite. La verticalité de l'écrit d'une enveloppe ou d'une couverture de livre n'a pas la même signification que celle des réécritures extralinéaires du brouillon. Pour interpréter la verticalité, il faut la rapporter à la pratique discursive dans laquelle elle s'inscrit, ici la préparation écrite d'un autre discours.

15. À cette différence importante près que l'interprétation génétique consiste à convertir les coordonnées spatiales du brouillon en coordonnées temporelles pour reconstruire le processus de production. Il s'agit d'une sémiotique indiciaire (Lebrave 1999). Mais de manière plus générale, l'espace de l'écrit est apte à instituer d'autres sémiotiques, tout à fait arbitraires. 
Benveniste Émile (2012), Dernières leçons. Collège de France (1968-1969), Paris, Gallimard/Seuil. Berrendonner Alain (2004), «Grammaire de l'écrit vs grammaire de l'oral : le jeu des composantes micro- et macro-syntaxiques », dans A. Rabatel (dir.), Interactions orales en contexte didactique, Lyon, PUL, p. 249-262.

Blanche-Benveniste Claire et alii (1990), Le Français parlé. Études grammaticales, Paris, Éditions du CNRS.

Blanche-Benveniste Claire, Caddeo Sandrine (2000), « Préliminaires à une étude de l'apposition dans la langue parlée », Langue française, $\mathrm{n}^{\circ} 125$, p. 60-70.

Blanche-Benveniste Claire, Jeanjean Colette (1987), Le Français parlé. Transcription et édition, Paris, Didier Érudition.

Caporossi Gilles G., Leblay Christophe (2014), « Introduction aux données temporelles de l'écriture », dans C. Leblay et G. Caporossi (dir.), Temps de l'écriture. Enregistrements et représentations, Louvain-la-Neuve, Academia/L'Harmattan, p. 5-15.

Coltier Danielle, Turco Gilbert (1988), « Des agents doubles de l'organisation textuelle, les marqueurs d'intégration linéaire », Pratiques, n 57 , p. 57-79.

Combe Dominique (2002), «L'ajout en rhétorique et poétique », dans Figures d'ajout. Phrase, texte, écriture, dir. J. Authier-Revuz et M.-C. Lala, Paris, Presses de la Sorbonne Nouvelle, p. 15-27.

Courtès Joseph (1995), Du lisible au visible : analyse sémiotique d'une nouvelle de Maupassant, d'une bande dessinée de B. Rabier, Louvain-la-Neuve, De Boeck.

DAusse François (2003), «Acte d'écriture, de lecture et ponctuation », dans Dynamique de l'écriture : approches pluridisciplinaires, dir. J.-P. Jaffré, Faits de Langues, n ${ }^{\circ} 22$, p. 223-230.

FAVRIAUD Michel (2011), «Plurisystème ponctuationnel, dimension, intensité des signes et architecturation du texte poétique », Langue française, $\mathrm{n}^{\circ} 172$, p. 83-98.

GADET Françoise (1996), «Une distinction bien fragile : oral/écrit », Tranel, n² 25, p. 13-27.

Galbraith David, Ford Sheila, Walker Gillian, Ford Jessica (2005), « The Contribution of Different Components of Working Memory to Knowledge Transformation during Writing », dans LI Educational Studies in Language and Literature, $\mathrm{n}^{\circ}$ 5, p. 113-145.

Gardes Tamine Joëlle (2004), Pour une grammaire de l'écrit, Paris, Belin.

Genette Gérard (1972), Figures III, Paris, Éditions du Seuil.

Goody Jack (1979 [1977]), La Raison graphique. La domestication de la pensée sauvage, Paris, Minuit.

Greimas Algirdas Julien (1983), « La soupe au pistou ou la construction d'un objet de valeur », dans Du Sens II, Paris, Éditions du Seuil, p. 157-169.

GrÉsILlon Almuth (1994), Éléments de critique génétique. Lire les manuscrits modernes, Paris, PUF.

Grésillon Almuth, Lebrave Jean-Louis, Viollet Catherine (1990), Proust à la lettre. Les intermittences de l'écriture, Tusson, Du Lérot.

Harris Roy (1993), La Sémiologie de l'écriture, Paris, Éditions du CNRS.

JAKOBSON Roman (1963 [1960]), «Linguistique et poétique », dans Essais de linguistique générale. 1 . Les fondations du langage, Paris, Minuit, p. 209-248.

Koch Peter, Oesterreicher Wulf (2001), «Langage oral et langage écrit », dans Lexikon der romanistischen Linguistik, t. I, Tübingen, Niemeyer, p. 584-627.

LeBrave Jean-Louis (1983), « Lecture et analyse du brouillon », Langages, ${ }^{\circ}$ 69, p. 11-23.

- 1987, Le Jeu de l'énonciation en allemand d'après les variantes manuscrites des brouillons de H. Heine, Thèse pour l'obtention du doctorat d'État présentée par Jean-Louis Lebrave sous la direction de M. Paul Valentin, Université Paris IV (4 vol.).

— 1992, «La critique génétique : une discipline nouvelle ou un avatar de la philologie », Genesis, $\mathrm{n}^{\circ} 1$, p. 33-72.

— 1999, «Les manuscrits entre la substance de l'expression et la substance du contenu », dans Catégories et connexions, dir. C. Cortès et A. Rousseau, Lille, Presses universitaires du Septentrion, p. 371-378. 
— 2006, « Du visible au lisible : comment représenter la genèse ? », Genesis, n 27, p. 11-18.

LEFEBVRE Julie (2011), «L'appel-renvoi de note comme observatoire de l'interpénétration des ponctuations blanche et noire », Langue française, $\mathrm{n}^{\circ} 172$, p. 69-82.

LugRIn Gilles (2006), Généricité et intertextualité dans le discours publicitaire de presse écrite, Berne, Peter Lang.

MAHrer Rudolf (2009), «De la textualité des brouillons. Prolégomènes à un dialogue entre linguistique et génétique des textes », Modèles linguistiques, t. XXX, vol. 59, dir. J.-M. Adam et I. Fenoglio, p. 51-70.

MAHrer Rudolf, Nicollier Valentine (2014), « Les brouillons font-ils texte ? Le cas des plans prérédactionnels de C.F. Ramuz », dans Faire texte. Unité(s) et (dis)continuité, dir. J.-M. Adam, Besançon, Presses universitaires de Franche-Comté.

Olive Thierry, Passerault Jean-Michel, Lebrave Jean-Louis, Le Bigot Nathalie (2010), « La dimension visuo-spatiale de la production de textes. Approche de psychologie cognitive et de critique génétique », Langages, ${ }^{\circ} 117$, p. 33-59.

Montandon Alain (dir.) (1990), Iconotextes, Paris, Ophrys.

Rey-Debove Josette (1988), «À la recherche de la distinction oral/écrit », dans Pour une théorie de la langue écrite, dir. N. Catach, Paris, Éditions du CNRS, p. 77-90.

SAussure Ferdinand de (1916), Cours de linguistique générale, Paris, Payot.

Testenoire Pierre-Yves (2010), « Genèse d'un principe saussurien : la linéarité », Revue Recto/Verso, $\mathrm{n}^{\circ} 6$, septembre (<www.revuerectoverso.com/IMG/pdf/Saussure.pdf $>$ ).

Vigner Gérard (1990), «Un type de texte : le dire de faire. Programmation d'actions et distribution du lexique », Pratiques, ${ }^{\circ}$ 66, p. 107-124. 
RUdOLF MAHrer est maître-assistant en linguistique française à l'Université de Lausanne et responsable du séminaire « Manuscrit - Linguistique - Cognition » de l'ITEM (CNRS-ENS). Ses recherches portent sur l'énonciation écrite notamment sous l'angle de ce qui apparente et différencie celle-ci de l'énonciation orale.

rudolf.mahrer@unil.ch

Résumés

\section{Écrire et parler. Quelques préalables théoriques}

L'écrit est utile à la préparation de l'oral. Cet article se propose d'éclairer cette évidence sous un angle sémiologique. À cette fin, nous nous focalisons ici sur les propriétés spécifiques des signaux des deux pratiques symboliques en jeu : processuelles et acoustiques du côté de l'oral, qui représente en l'occurrence la pratique préparée, statiques et graphiques, du côté de la pratique préparante. Pour comprendre comment les qualités de la substance graphique disposent à la pratique de la préparation du discours, nous considérons le rapport de l'écrit et de l'oral au temps et à l'espace, mais aussi à la supposée linéarité du signifiant. Pour décrire comment l'espace graphique prépare efficacement le temps de la performance orale, il faut aussi préciser ce qu'on peut entendre par préparation du discours. Nous finirons par quelques hypothèses à ce propos, entre rhétorique classique et psycholinguistique.

The written text is useful for preparing the oral. This article sets out to elucidate this evidence from a semiological perspective. To this end we will focus on the specific attributes of the signals of the two symbolic practices at play: processual and acoustical on the oral side, which represents in other words the prepared practice, and static and graphic on the preparing practice side. To understand how the graphic substance's qualities induce the practice of speech preparation, we will consider the written and oral's relationship with time and space, but also with the assumed linearity of the signifying. To describe how the graphic space efficiently prepares the time of the oral performance, we should also explain what is meant by speech preparation. We will conclude with a few hypotheses in this regard, between classical rhetoric and psycholinguistics.

Das Schriftliche dient zur Vorbereitung des Mündlichen. In diesem Artikel soll diese Tatsache aus einem semiologischen Blickwinkel beleuchtet werden. Dazu konzentrieren wir uns auf die spezifischen Eigenschaften der Zeichen von zwei entsprechenden symbolischen Praktiken: prozessuale und akustische auf der Seite des Mündlichen, welches in dem Fall die vorbereitete Praxis darstellt, statisch und graphisch auf der Seite der vorbereitenden Praxis. Um zu verstehen, wie die Qualitäten der graphischen Substanz der Praxis der Redevorbereitung dienen, betrachten wir den Zusammenhang des Schriftlichen und des Mündlichen mit Zeit und Raum, aber auch mit der angenommenen Linearität des Signifikanten. Um zu beschreiben, wie der graphische Raum effizient die Zeit der mündlichen Performanz vorbereitet, muss jedoch auch erklärt werden, was unter Redevorbereitung verstanden werden kann. Der Artikel schließt mit einigen Hypothesen diesbezüglich, zwischen der klassischen Rhetorik und der Psycholinguistik.
E1 texto escrito es útil para la preparación del discurso oral. Este artículo se propone considerar esta evidencia en una perspectiva semiológica. Con este objetivo, focalizaremos aquí las propiedades específicas de las señales de dos prácticas simbólicas en juego: procesales y acústicas, en lo que respecta lo oral, que representa en este caso la práctica preparada, estática y gráfica, en lo que respecta la práctica preparatoria. Para entender cómo las cualidades de la sustancia gráfica predisponen a la práctica de la preparación del discurso, consideraremos la relación que vincula lo escrito y lo oral con el tiempo y el espacio, pero también con la supuesta linealidad del significante. Para describir cómo el espacio gráfico prepara eficazmente el tiempo de la performance oral, es necesario precisar lo que es posible entender por preparación del discurso. Concluiremos con algunas hipótesis al respecto, entre retórica clásica y psicolingüística.

$\mathbf{O}$ escrito é útil na preparação do oral. Este artigo propõe-se iluminar essa evidência de um ângulo semiológico. Focamos para isso as propriedades específicas dos sinais das duas práticas simbólicas implicadas: procedimentais e acústicas no plano oral, que representa a prática preparada; estáticas e gráficas enquanto prática preparante. Para entender como as qualidades da substância gráfica dispõem à prática da preparação do discurso, recorremos à relação entre escrito e oral no tempo e no espaço, mas também à chamada linearidade do significante. Para descrever como o espaço gráfico acomoda o tempo da realização oral, devemos também orecisar o que se entende por preparação do discurso. Formularemos a concluir algumas hipóteses, de natureza retórica tradicional e psicolinguística.

Lo scritto è utile alla preparazione dell'orale. Quest'articolo si propone di chiarire quest'evidenza da un punto di vista semiologico. Per far ciò, ci concentreremo sulle proprietà specifiche dei segnali delle due pratiche simboliche in gioco: processuali e acustici riguardo all'orale (che rappresenta in questo caso la pratica preparata), statici e grafici riguardo alla pratica preparante. Per comprendere in che modo le qualità della sostanza grafica predispongono alla pratica della preparazione del discorso, considereremo il rapporto dello scritto e dell'orale con il tempo e con lo spazio, ma anche con la supposta linearità del significante. Per descrivere in che modo lo spazio grafico prepara efficacemente il tempo della performance orale, bisogna anche precisare ciò che s'intende per preparazione del discorso; l'articolo terminerà con qualche ipotesi a riguardo, tra retorica classica e psicolinguistica. 